\title{
Chemical Tools for Probing Histone Deacetylase (HDAC) Activity
}

\author{
Masafumi MinoshImA*,** and Kazuya KIKUCHI $* * * * \dagger$ \\ *Graduate School of Engineering, Osaka University, 2-1 Yamadaoka, Suita, Osaka 565-0871, Japan \\ **Institute of Academic Initiatives, Osaka University, 2-1 Yamadaoka, Suita, Osaka 565-0871, Japan \\ ***Immunology Frontier Research Center (IFReC), Osaka University, 2-1 Yamadaoka, Suita, Osaka 565-0871, \\ Japan
}

\begin{abstract}
Histone deacetylases (HDACs) enzymes are responsible for removing epigenetic markers on histone proteins, which results in chromatin inactivation and gene repression. An evaluation of HDAC activity is essential for not only determining the physiological function of HDACs, but also for developing HDAC-targeting drugs. This review focuses on the chemical tools used to detect HDAC activity. We highlight activity-based probes and positron emission tomography probes based on the chemical structure of the inhibitors. We also summarize fluorogenic probes used in single-step methods for HDAC detection. These fluorogenic probes are designed based on the nucleophilicity of the amino group, aggregation via electrostatic interactions, and changes in the DNA binding properties. These fluorogenic systems may enable facile and rapid screening to evaluate HDAC inhibitors, which will contribute to the development of epigenetic drugs.
\end{abstract}

Keywords Histone deacetylase, fluorogenic probes, activity-based probes, positron emission tomography

(Received November 28, 2014; Accepted January 5, 2015; Published April 10, 2015)

\begin{tabular}{llll}
\hline Introduction & 287 & 5 Conclusions & 291 \\
2 Activity-based Probes & 288 & 6 Acknowledgements & 291 \\
3 PET Probes & 289 & 7 References & 291 \\
4 Fluorogenic Probes & 290 & & \\
\hline
\end{tabular}

\section{Introduction}

In eukaryotic cells, DNA is compactly wrapped inside the nucleus, forming chromatin structures that interact with nuclear histone proteins. DNA and dimers of four histone proteins $(\mathrm{H} 2 \mathrm{~A}, \mathrm{H} 2 \mathrm{~B}, \mathrm{H} 3$, and $\mathrm{H} 4)$ form a nucleosome, the structural unit of chromatin. ${ }^{1}$ Histones are subjected to chemical modifications, such as acetylation, methylation, and phosphorylation after translation..$^{2,3}$ Histone modification states are reversibly and dynamically controlled by numerous enzymes.

The acetylation of histones is an essential post-translational modification associated with epigenetic gene regulation. In 1964, Allefrey et al. demonstrated the relationship between the histone acetylation level and the transcriptional activity in cells. ${ }^{4}$ Acetylation occurs on the $\varepsilon-\mathrm{NH}_{2}$ groups of lysine residues of

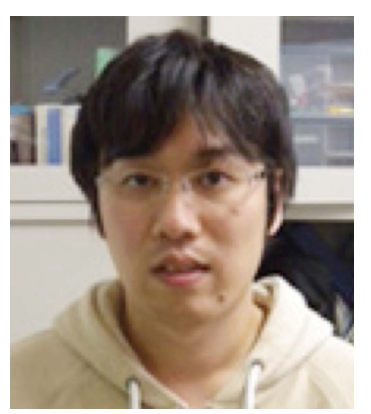

Masafumi Minoshima received his Ph.D. in 2010 from Kyoto University. He worked at the University of Tokyo as a Specially-appointed Assistant Professor (2010 - 2012), and then moved to Osaka University to work as a Speciallyappointed Assistant Professor of the Institute of Academic Initiatives (2012 -). His current research interest is the development of chemical probes for the elucidation of epigenetic function.

† To whom correspondence should be addressed.

E-mail: kkikuchi@mls.eng.osaka-u.ac.jp

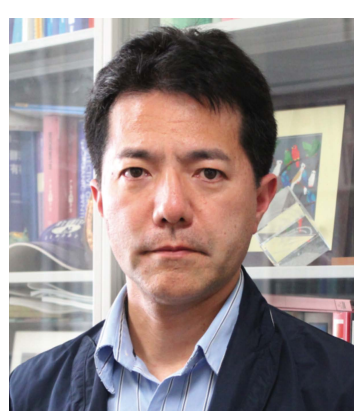

Kazuya KIKUCHI graduated from the University of Tokyo and did his postdoctoral training in the US at the University of California, San Diego (UCSD) with Prof. Roger Y. Tsien and the Scripps Research Institute with Prof Donald Hilvert. He was appointed as a Research Associate at the University of Tokyo thereafter and promoted to Associate Professor. He was appointed as a Professor at Osaka University in 2005 . During this period he became involved in molecular imaging probe development for both fluorescence imaging and magnetic resonance imaging. His research interest focuses on both in vivo imaging and single molecule cellular imaging. 

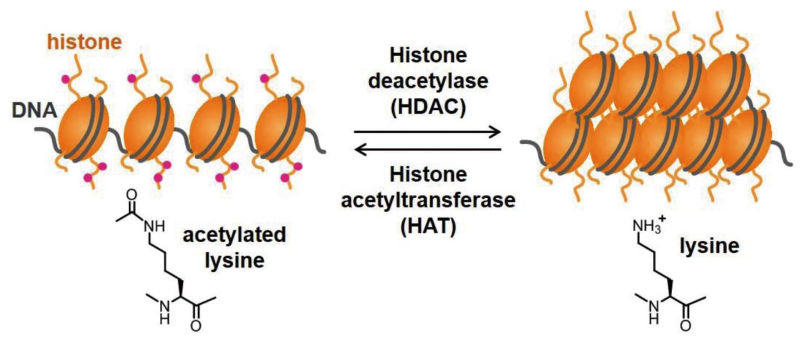

Fig. 1 Regulation of the chromatin structure by the acetylation state of histones. The magenta circles indicate acetylation of the histone tail. HAT and HDAC modify lysine residues.

histones through the action of histone acetyltransferases (HATs), ${ }^{5}$ while the acetyl group is removed by histone deacetylases (HDACs) (Fig. 1). ${ }^{6}$ Histone acetylation locally activates chromatin structures, making the structure more accessible to transcriptional factors and RNA polymerases, and resulting in transcriptional activation. In contrast, the deacetylation of histones leads to the formation of condensed chromatin structures, suppressing gene expression. Since Schreiber et al. first identified HDAC, ${ }^{6} 18$ HDACs have been identified in humans. HDACs are classified into four groups, including class I (HDAC1, 2, 3, 8, 10), class II (HDAC4, 5, 6, 7, 9), class III (SIRT1 - 7, also known as sirtuins), and class IV (HDAC11). Class I, II, and IV HDACs are metallohydrolyases that use $\mathrm{Zn}^{2+}$ in their active sites. ${ }^{7}$ The active site contains a zinc-binding site and charge-relay systems composed of asparagine and histidine residues. ${ }^{8}$ Class III HDACs catalyze deacetylation using a nicotinamide adenine dinucleotide $\left(\mathrm{NAD}^{+}\right)$ as a cofactor. ${ }^{9,10}$ Recent reports found that several class III HDACs remove non-acetyl groups from proteins. For example, SIRT5 reacts with succinylated, malonylated, and glutamylated lysine residues, while SIRT6 mainly reacts with fatty acylated lysine residues with long alkyl chains. ${ }^{11,12}$ These HDACs are present in the nucleus, cytosol, and mitochondria. Nuclear HDACs, such as most zinc-dependent HDACs and some sirtuins, mainly deacetylate histone proteins, while cytosolic and mitochondrial HDACs can deacetylate non-histone proteins.

The physiological roles of HDACs include roles in development and differentiation, which depends on the specific tissues and extracellular environment. ${ }^{13}$ Class III HDACs are also associated with metabolic process, since their activities depend on the amount of the cofactor $\mathrm{NAD}^{+}$available. ${ }^{10}$ In addition, aberrant gene expression resulting from an altered histone acetylation level is associated with cancer, neurodegenerative diseases, and immune diseases. ${ }^{14-19}$ For example, several cancer-suppression genes, such as cyclin-dependent kinase inhibitor p21, are down-regulated by local chromatin inactivation in malignant cells. ${ }^{19}$ The inhibition of HDACs causes transcriptional activation of cancer-suppressor genes, resulting in cell-cycle arrest and apoptosis induction in malignant cells. Therefore, probing HDAC activity is essential not only for understanding physiological HDAC modulation, but also for evaluating inhibitors during drug development.

In this review, we highlight the progress in chemical tools and methods used for investigating HDAC activity. Although antibody-based techniques, such as western blotting-, enzymelinked immunosorbent assay-, and chromatin immunoprecipitation (ChIP)-based analyses are also powerful tools for detecting histone acetylation levels, these methods highly depend on the quality of the antibodies. ${ }^{20}$ In addition, antibodies are cell impermeable and often utilized for staining of fixed cells, which has some limitation in understanding HDAC dynamics in living cells. Mass spectra-based proteomic approaches are also useful for determining substrate specificity and quantifying HDAC activity. ${ }^{21-25}$ In this review, we mainly focus on HDAC detection methods using chemical probes with positron emission tomography (PET) and fluorescence that can be expanded for imaging applications.

\section{Activity-based Probes}

Activity-based protein profiling methods are interesting approaches for detecting enzymes and their substrates. Activitybased probes are covalently directed to enzyme active sites for chemical labeling of targeted enzymes. ${ }^{26}$ These probes generally consist of a warhead domain, a covalent enzyme inhibitor, and a tag domain for further chemical labeling. The alkyne tag is mainly used for subsequent coupling with azide-conjugating biotin or a fluorophore via $\mathrm{Cu}(\mathrm{I})$-catalyzed azide-alkyne cycloaddition. The biotinylated protein complexes can be purified using affinity beads immobilized to streptavidin, which can be subjected to further proteomic analysis, such as in liquid chromatography and tandem mass spectrometry (LC-MS/MS). The trapped proteins can be precisely identified in purified samples. Fluorophore-labeled protein complexes can be detected with high sensitivity on gels and in test tubes. This strategy has been utilized to detect various types of enzymes, including serine proteases, cysteine proteases, kinases, and acetyltransferases. ${ }^{27-30}$

Similar to the enzymes described above, activity-based probes for zinc-dependent HDACs have been designed based on the chemical structure of the inhibitors. Salisbury et al. reported activity-based probes based on suberoylanilide hydroxamic acid (SAHA), a non-covalent HDAC inhibitor that chelates a zinc ion in the active site of zinc-dependent HDACs, conjugated with an alkyne tag and a benzophenone as a photocrosslinker (Fig. 2). ${ }^{31,32}$ Although SAHA is a reversible inhibitor, bound HDACs can be trapped by a photocrosslinking benzophenone moiety under UV irradiation. Crosslinked proteins, including HDAC1 and 2, were detected in HeLa cell lysates after labeling with rhodamine and subsequent fluorescence detection in gels. Moreover, this probe also selectively trapped other proteins, including methylated DNA binding protein 3 (MBD3) in cell lysates and living cells. This result suggests that these proteins may exist in close proximity to trapped HDACs and be associated with substrate recognition and activity. Proteomic analysis of trapped proteins in aggressive and non-aggressive melanoma cell lines revealed that the detection levels in HDAC6 and a larger HDAC complex CoREST were significantly altered compared to that in other cell lines, suggesting a cell dependency of the HDAC function. $\mathrm{Xu}$ et al. also developed activity-based probes based on the pimelic diphenylamide inhibitor. ${ }^{33}$ They found that the probe specifically targets HDAC 3 in cells, and that the role of HDAC3 in gene expression is associated with neurodegenerative Friedreich's ataxia. Recently, Diyabalanage et al. reported an alternative strategy for the detection of HDAC activity in living cells based on aldehyde trapping. ${ }^{34}$ They developed an activity based probe, HP-1, a fluorescent dye, 7-nitro-benzo-2-oxa-1,3diazole (NBD), conjugated with an enamide via an aliphatic linker. The terminal enamide can be deacetylated by HDAC, yielding a reactive aldehyde via hydrolysis of an enamine. Since aldehydes are crosslinked with amino groups of proteins, HDAC activity can be detected by the accumulation of the fluorescence trapped in the protein fraction. Using this strategy, 
(A)

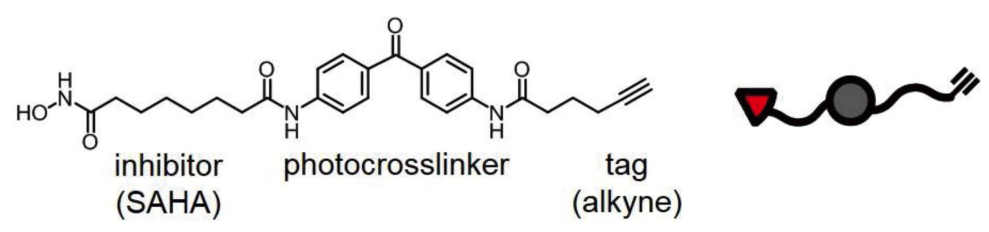

(B)

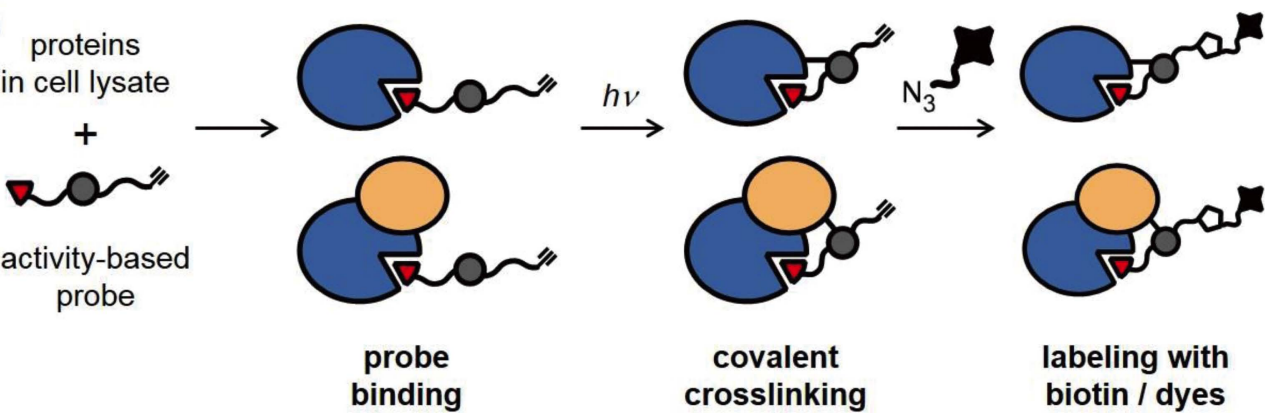

Fig. 2 Activity-based probes and their applications. (A) Chemical structure of an activity-based probe for HDAC activity based on suberoylanilide hydroxamic acid (SAHA). (B) Schematic illustration of the labeling of HDACs and interacting proteins for detection using an activity-based probe. Red triangles and gray circles show the inhibitor and photocrosslinker regions, respectively. Selective proteins including HDACs are bound to an inhibitor domain of the activity-based probe. After photoirradiation, proteins and probes are covalently crosslinked. Subsequent labeling with biotin or fluorescent dyes via click chemistry enables further purification and detection.

they showed an HDAC activity-dependent cellular accumulation of HP-1 by fluorescence imaging. Although further improvement on the reaction efficiency and selectivity will be required, this novel strategy can potentially be utilized in other imaging modalities, such as magnetic resonance imaging (MRI) and PET for in vivo studies.

\section{PET Probes}

PET is a useful method for in vivo imaging applications to investigate the detailed biodistribution of the exogenously administrated molecules with excellent sensitivity. ${ }^{35}$ The HDAC inhibitor, SAHA, and a microbial natural product, FK228 (Romidepsin), were recently approved by the FDA for the clinical treatment specific T-cell malignancies. Evaluations of the pharmacokinetic properties in vivo are urgently required for further development of HDAC inhibitors. For the development of PET probes, a radioisotope, such as fluorine- $18\left({ }^{18} \mathrm{~F}\right.$, half-life $\sim 110 \mathrm{~min}$ ) or carbon-11 ( ${ }^{11} \mathrm{C}$, half-life $\sim 20 \mathrm{~min}$ ), was introduced to the target molecules. Several PET-active probes based on HDAC inhibitors were developed for the detection of HDAC and biodistribution of HDAC inhibitors in vivo (Fig. 3). Reid et al. developed ${ }^{18} \mathrm{~F}$-modified SAHA $\left(\left[{ }^{18} \mathrm{~F}\right]\right.$ FAHA) for the targeting of HDAC, and evaluated the metabolic processes and pharmacokinetic properties involved. ${ }^{36}$ Injected $\left[{ }^{18} \mathrm{~F}\right]$ FAHA molecules are rapidly metabolized to $\left[{ }^{18} \mathrm{~F}\right]$ fluoroacetate, and can enter the baboon brain. However, this probe lacks the hydroxamic acid moiety that is essential for SAHA pharmacological activity. Hendricks et al. developed another ${ }^{18} \mathrm{~F}$-SAHA PET probe possessing a hydroxamic acid in order to address this problem..$^{37}$ This probe exhibited a similar potency and selectivity to SAHA, and showed a time-dependent accumulation in tumors in a murine ovarian cancer model. A benzamide-type of HDAC inhibitor, MS-275, showed potential for the clinical treatment of cancer and neurological diseases. However, $\left[{ }^{11} \mathrm{C}\right] \mathrm{MS}-275$ showed a very low blood-brain barrier (BBB) permeability based on PET imaging results. ${ }^{38}$ Thus,<smiles>O=C(CF)NCCCCCC(=O)Nc1ccccc1</smiles>

$\left[{ }^{18} \mathrm{~F}\right]$ FAHA<smiles>O=C(CCCCCCC(=O)Nc1ccc([18F])cc1)NO</smiles><smiles>Nc1ccccc1NC(=O)c1ccc(CNC(=O)OCc2cccnc2)cc1</smiles>

[11 C] MS-275<smiles>CN(C)Cc1ccc(C(=O)Nc2cc(-c3ccccc3)ccc2N)cc1</smiles>

$\left[{ }^{11} \mathrm{C}\right] \mathrm{MS}-275$ derivative

Fig. 3 Developed PET probes based on the chemical structures of HDAC inhibitors.

more active and BBB-permeable MS-275 derivatives were developed for further applications to neurological diseases. ${ }^{39}$ 
(A)

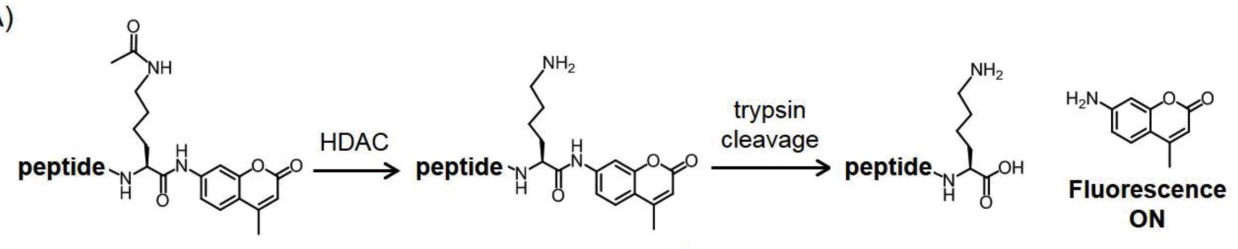

(B)

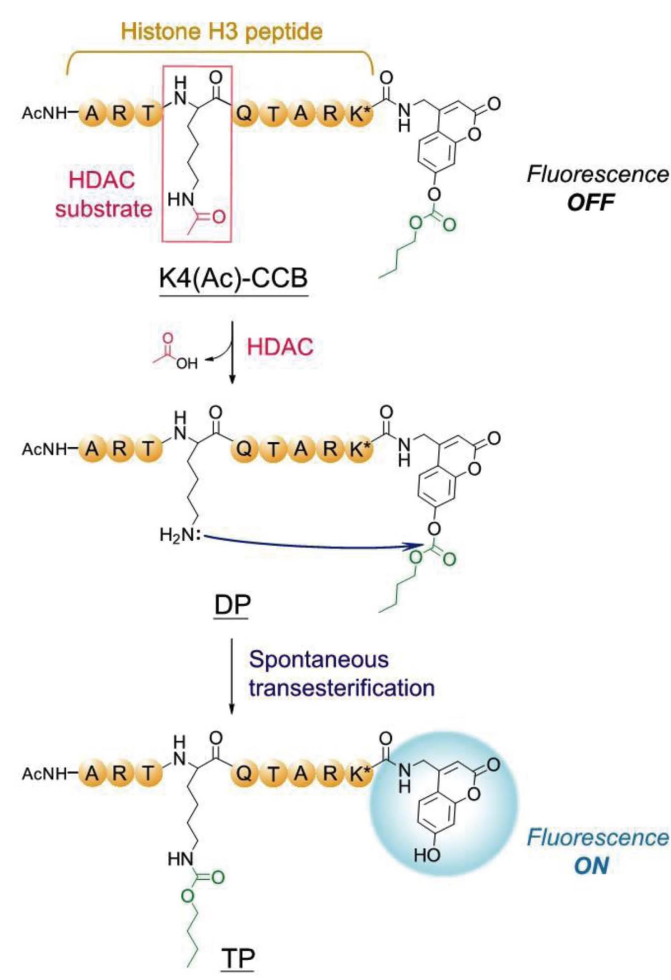

(C)
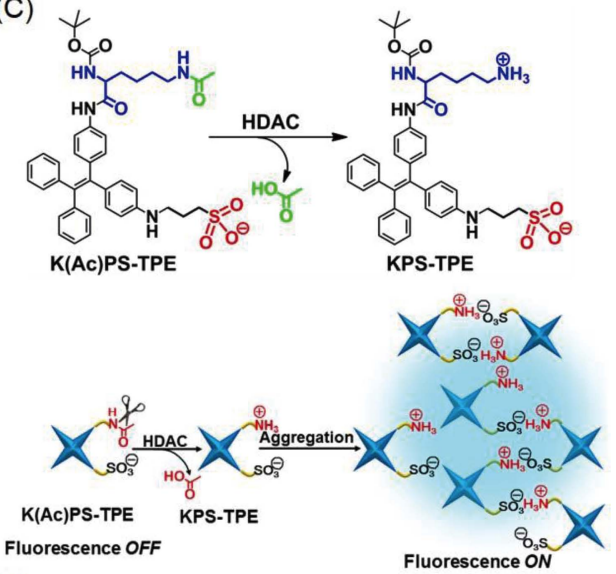

(D)

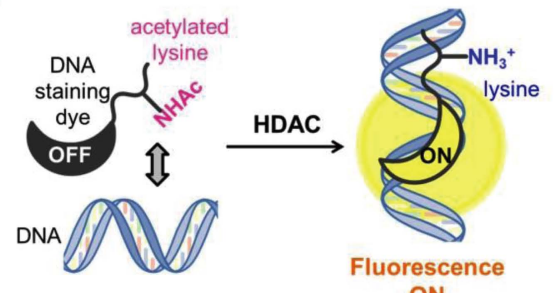

ON

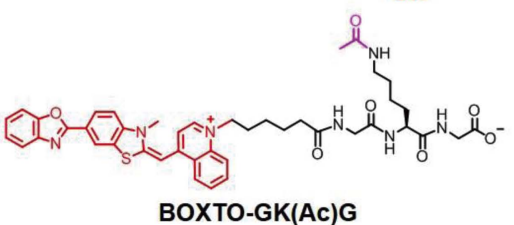

Fig. 4 Methods for detecting HDAC activity using fluorogenic probes. (A) Enzyme-coupled detection using a peptide-based fluorescent probe. Subsequent trypsin digestion is required for fluorescence enhancement. (B) Single-step HDAC detection system using a fluorogenic probe based on spontaneous transesterification. Deacetylated probe (DP) spontaneously produced the transferred probe (TP) via transesterification. (C) HDAC detection using aggregation-induced emission. Deacetylation of K(Ac)PS-TPE emits fluorescence upon aggregation through electrostatic interactions between cationic lysines and anionic sulfonates. (D) HDAC detection using the DNA binding dye BOXTO. The deacetylation of BOXTO-GK(Ac)G increases the DNA binding ability, resulting in the fluorescence enhancement. Reproduced with permission from Refs. 45 - 47.

\section{Fluorogenic Probes}

Fluorescence detection is a versatile method for examining enzymatic activity with high sensitivity. In particular, fluorescence-based detection systems are useful for drug screening because of they can be used in high-throughput applications. Because HDAC inhibitors with high potency or excellent selectivity are highly demanded as drugs for cancer and other diseases, considerable efforts have been made in the development of novel HDAC-inhibiting compounds using a mechanism-based approach. ${ }^{40}$ Fluorogenic probes that switch their fluorescence properties upon enzyme reactions are powerful tools in fluorescence detection systems. Fluorescent properties can be controlled based on the mechanism of Förster resonance energy transfer (FRET), which depends on the distance between a fluorophore and a quenching molecule. In contrast, photoinduced electron transfer, which depends on the electronic energy level between an electron donor and acceptor, is also utilized for fluorescence switching. Based on these fluorescence-switching mechanisms, a number of fluorescent probes have been developed and applied for detecting the activities of enzymes, such as proteases, glycosylases, and esterases. ${ }^{41,42}$ However, the design of fluorogenic probes for directly detecting HDACs is difficult because the mechanisms described above are not available for the deacetylation of aliphatic amines.

Using two-step enzyme-coupled reactions, Weggener et al. developed a fluorogenic method for detecting HDAC activity (Fig. 4A). ${ }^{43}$ They developed a peptide containing an acetylated lysine conjugated to an aminomethylcoumarin (AMC) at the C-terminus. An AMC moiety shows little fluorescence when 
acylated at the 7-amino group. After deacetylation of the probe, the peptide bond between the C-terminal of deacetylated lysine and the 7-amino group of AMC can be cleaved by trypsin, an endoprotease that cleaves the C-terminus of basic amino acids. Trypsin cleavage results in the release of AMC and an increase of fluorescence. This method can be used to evaluate the activity of various HDACs as well as the substrate specificity. However, the initial probe shows a limitation in the detection of class IIa HDACs. Brandner et al. reported that introduction of a relatively unstable trifluoroacetylated lysine in place of the acetylated lysine enabled efficient detection of class IIa HDACs, which can include all $\mathrm{Zn}^{2+}$-dependent HDACs. ${ }^{44}$ A systematic evaluation of the isoform selectivity of the inhibitors enabled the identification of a novel HDAC inhibitor with broad HDAC specificity. Such peptide-based fluorogenic probes are commercially available as Fluor-de-Lys ${ }^{\circledR}$ probes for HDAC detection. However, one limitation of the probe is the inflexibility of the probe design, since the fluorophore (AMC) should be located at the adjacent position of the acetylated lysine residue. Moreover, an additional protease digestion step is required to enhance the fluorescence after the HDAC reaction. For high-throughput screening of HDAC inhibitors, more facile and direct detection methods are required.

Recently, our group developed novel fluorogenic probes that do not require additional enzyme treatment for HDAC detection..$^{45}$ One such approach is based on the nucleophilicity of the amino group of deacetylated lysine (Fig. 4B). Based on this concept, a fluorogenic probe $\mathrm{K} 4(\mathrm{Ac}) \mathrm{CCB}$ was designed by conjugating a 7-acylated hydroxycoumarin dye possessing an electrophilic carbonate linker to a substrate peptide derived from residues $1-9$ of histone $\mathrm{H} 3$. Upon probe deacetylation, the nucleophilic aliphatic amine at the lysine residue intramolecularly attacks the carbonate linker, producing fluorescent aminocoumarin. The intramolecular transesterification from a 7-hydroxycoumarin to a deacetylated lysine was spontaneously observed after deacetylation by HDAC using high-performance liquid chromatography analysis. Based on this finding, the activity of SIRT1 deacetylase was evaluated based on a fluorescence increase, which demonstrated the usefulness of single-step HDAC detection. Another approach was based on aggregation-induced emission, which enhances fluorescence by a conformational restriction of the molecules in the aggregated state (Fig. 4C). ${ }^{46}$ A tetraphenylethylene-derived fluorogenic probe, $\mathrm{K}(\mathrm{Ac})$-PS-TPE, possessing an acetyllysine and a sulfonate group, was also developed. Deacetylation of acetyllysine causes aggregation-induced emission because of the changes in the electrostatic interactions between the cationic $\mathrm{N}-\varepsilon$-ammonium ion and the sulfonate group. Very recently, we also developed a fluorogenic detection method based on a DNA binding dye that increases the fluorescence upon binding to DNA (Fig. 4D). ${ }^{47}$ This strategy is directed to the detection of HDAC activity using DNA in the nucleus, where most of the HDACs exist. A fluorogenic probe was developed by the conjugation of the DNA binding BOXTO dye to a peptide containing an acetylated lysine. Deacetylation of the probe markedly increased the DNA binding affinity because of electrostatic interactions between a cationic lysine residue and phosphate backbone in DNA. As a result, a fluorescence enhancement by 7.8-fold was observed upon deacetylation by HDAC in the presence of an excess amount of purified DNA. This system enabled real-time monitoring of the HDAC activity and a rapid evaluation of the inhibitor potencies with a one-pot procedure. Although the cellular HDAC activity has not been successfully demonstrated, further improvements of the cell permeability and the reaction efficiency of the probe will dynamically monitor HDAC activity in living cells. These facile and direct methods can be used to evaluate the enzyme activity and inhibitor potency in a one-step procedure, which is applicable to the development of drugs targeting histonemodifying enzymes.

\section{Conclusions}

In this review, we described methods for detecting HDAC activity, while mainly focusing on chemical approaches, including PET and fluorescence detection. Although these fluorogenic detection methods facilitate rapid evaluations of HDAC-targeting inhibitors for drug development studies, elucidating the dynamics of HDAC activity in living cells remains challenging. Genetically encoded fluorescent protein sensors for HDAC detection were reported for the evaluation of cellular HDAC activity based on FRET. ${ }^{48}$ However, fluorogenic chemical probes available for live cell imaging of HDAC activity will also become attractive tools because of the facile control of the timing using chemical probes, making them suitable for understanding time- or cell-dependent HDAC activity. Further advances in the design of chemical probes for HDAC detection based on both chemistry and biology will contribute to the development of epigenetic studies and medical applications.

\section{Acknowledgements}

This work was supported by the Japan Science and Technology Agency (JST), the Ministry of Education, Culture, Sports, Science and Technology (MEXT) of Japan (Grants 24108724, 25220207, 25242072, and 25620133 to K. K. 26750371 to M. M.), the Funding Program for World-Leading Innovative R\&D on Science and Technology from the Japan Society for the Promotion of Science (JSPS), CREST from JST, and the Asahi Glass Foundation.

\section{References}

1. K. Luger, A. W. Mäder, R. K. Richmond, D. F. Sargent, and T. J. Richmond, Nature, 1997, 389, 251.

2. B. Strahl and C. D. Allis, Nature, 2000, 403, 41.

3. T. Jenuwein and C. D. Allis, Science, 2001, 293, 1074.

4. V. G. Allefrey, R. Faulkner, and A. E. Mirsky, Proc. Natl. Acad. Sci. U. S. A., 1964, 51, 786.

5. J. Brownell, J. Zhou, T. Ranalli, R. Kobayashi, D. Edmondson, S. Roth, and C. D. Allis, Cell, 1996, 84, 843.

6. J. Taunton, C. Hassig, and S. A. Schreiber, Science, 1996, $272,408$.

7. X.-J. J. Yang and E. Seto, Nat. Rev. Mol. Cell Biol., 2008, 9, 206.

8. M. Finnin, J. Donigian, A. Cohen, V. Richon, R. Rifkind, P. Marks, R. Breslow, and N. Pavletich, Nature, 1999, 401, 188.

9. S. Imai, C. Armstrong, M. Kaeberlein, and L. Guarente, Nature, 2000, 403, 795.

10. M. C. Haigis and L. P. Guarente, Genes Dev, 2006, 20, 2913.

11. J. Du, Y. Zhou, X. Su, J. J. Yu, S. Khan, H. Jiang, J. Kim, J. Woo, J. H. Kim, B. H. Choi, B. He, W. Chen, S. Zhang, R. A. Cerione, J. Auwerx, Q. Hao, and H. Lin, Science, 2011, $334,806$.

12. H. Jiang, S. Khan, Y. Wang, G. Charron, B. He, C. 
Sebastian, J. Du, R. Kim, E. Ge, R. Mostoslavsky, H. C. Hang, Q. Hao, and H. Lin, Nature, 2013, 496, 110.

13. M. Haberland, R. L. Montgomery, and E. N. Olson, Nat. Rev. Genet., 2009, 10, 32.

14. P. Marks, R. Rifkind, V. Richon, R. Breslow, T. Miller, and W. Kelly, Nat. Rev. Cancer, 2001, 1, 194.

15. J. E. Bolden, M. J. Peart, and R. W. Johnstone, Nat. Rev. Drug Discovery, 2006, 5, 769.

16. D. Herman, K. Jenssen, R. Burnett, E. Soragni, S. L. Perlman, and J. M. Gottesfeld, Nat. Chem. Biol., 2006, 2, 551.

17. T. Abel and S. R. Zukin, Curr. Opin. Pharmacol., 2008, 8, 57.

18. R. Tao, E. F. de Zoeten, E. Ozkaynak, C. Chen, L. Wang, P. M. Porrett, B. Li, L. A. Turka, E. N. Olson, M. I. Greene, A. D. Wells, and W. W. Hancock, Nat. Med., 2007, 13, 1299.

19. Y. Sowa, T. Orita, S. Minamikawa, K. Nakano, T. Mizuno, H. Nomura, and T. Sakai, Biochem. Biophys. Res. Commun., 1997, 241, 142.

20. T. Egelhofer, A. Minoda, S. Klugman, K. Lee, P. KolasinskaZwierz, A. Alekseyenko, M.-S. Cheung, D. Day, S. Gadel, A. A. Gorchakov, T. Gu, P. V. Kharchenko, S. Kuan, I. Latorre, D. Linder-Basso, Y. Luu, Q. Ngo, M. Perry, A. Rechtsteiner, N. C. Riddle, Y. B. Schwartz, G. A. Shanower, A. Vielle, J. Ahringer, S. C. Elgin, M. I. Kuroda, V. Pirrotta, B. Ren, S. Strome, P. J. Park, G. H. Karpen, R. D. Hawkins, and J. D. Lieb, Nat. Struct. Mol. Biol., 2011, 18, 91.

21. B. Garcia, S. Mollah, B. Ueberheide, S. Busby, T. Muratore, J. Shabanowitz, and D. Hunt, Nat. Protocols, 2007, 2, 933.

22. H. Eberl, M. Mann, and M. Vermeulen, ChemBioChem, 2011, 12, 224.

23. A. E. Merrill and J. J. Coon, Curr. Opin. Chem. Biol., 2013, 17,779 .

24. M. Bantscheff, C. Hopf, M. Savitski, A. Dittmann, P. Grandi, A.-M. Michon, J. Schlegl, Y. Abraham, I. Becher, G. Bergamini, M. Boesche, M. Delling, B. Dümpelfeld, D. Eberhard, C. Huthmacher, T. Mathieson, D. Poeckel, V. Reader, K. Struck, G. Sweetman, U. Kruse, G. Neubauer, N. G. Ramsden, and G. Drewes, Nat. Biotechnol., 2011, 29, 255.

25. Y. Chen, W. Zhao, J. Yang, Z. Cheng, H. Luo, Z. Lu, M. Tan, W. Gu, and Y. Zhao, Mol. Cell. Proteomics, 2012, 11, 1048.

26. B. F. Cravatt, A. T. Wright, and J. W. Kozarich, Annu. Rev. Biochem., 2008, 77, 383.

27. N. Jessani, M. Humphrey, W. H. McDonald, S. Niessen, K. Masuda, B. Gangadharan, J. R. Yates III, B. M. Mueller, and B. F. Cravatt., Proc. Natl. Acad. Sci. U. S. A., 2004,
$101,13756$.

28. D. Greenbaum, K. F. Medzihradszky, A. Burlingame, and M. Bogyo, Chem. Biol., 2000, 7, 569.

29. M. S. Cohen, C. Zhang, K. M. Shokat, and J. Taunton, Science, 2005, 308, 1318.

30. M. Yu, L. de Carvalho, G. Sun, and J. Blanchard, J. Am. Chem. Soc., 2006, 128, 15356.

31. C. Salisbury and B. F. Cravatt, Proc. Natl. Acad. Sci. U. S. A., 2007, 104, 1171.

32. C. Salisbury and B. F. Cravatt, J. Am. Chem. Soc., 2008, 130, 2184.

33. C. Xu, E. Soragni, C. Chou, D. Herman, H. Plasterer, J. R. Rusche, and J. M. Gottesfeld, Chem. Biol., 2009, 16, 980.

34. H. V. K. Diyabalanage, G. C. V. Bittner, E. L. Ricq, and J. M. Hooker, ACS Chem. Biol., 2014, 9, 1257.

35. M. E. Phelps, Proc. Natl. Acad. Sci. U. S. A., 2000, 97, 9226.

36. A. Reid, J. Hooker, E. Shumay, J. Logan, C. Shea, S. Kim, S. Collins, Y. Xu, N. Volkow, and J. Fowler, Nucl. Med. Biol., 2009, 36, 247.

37. J. A. Hendricks, E. J. Keliher, B. Marinelli, T. Reiner, R. Weissleder, and R. Mazitschek, J. Med. Chem., 2011, 54, 5576.

38. J. Hooker, S. Kim, D. Alexoff, Y. Xu, C. Shea, A. Reid, N. Volkow, and J. Fowler, ACS Chem. Neurosci., 2010, 1, 65.

39. Y. Seo, Y. Kang, L. Muench, A. Reid, S. Caesar, L. Jean, F. Wagner, E. Holson, S. Haggarty, P. Weiss, P. King, P. Carter, N. D. Volkow, J. S. Fowler, J. M. Hooker, and S. W. Kim, ACS Chem. Neurosci., 2014, 5, 588.

40. A. Bieliauskas and M. Pflum, Chem. Soc. Rev., 2008, 37, 1402.

41. P. R. Selvin, Nat. Struct. Mol. Biol., 2000, 7, 730.

42. A. P. de Silva, H. Q. N. Gunaratne, T. Gunnlaugsson, A. J. M. Huxley, C. P. McCoy, J. T. Rademacher, and T. E. Rice, Chem. Rev., 1997, 97, 1515.

43. D. Wegener, F. Wirsching, D. Riester, and A. Schwienhorst, Chem. Biol., 2003, 10, 61.

44. J. Bradner, N. West, M. Grachan, E. Greenberg, S. Haggarty, T. Warnow, and R. Mazitschek, Nat. Chem. Biol., 2010, 6, 238.

45. R. Baba, Y. Hori, S. Mizukami, and K. Kikuchi, J. Am. Chem. Soc., 2012, 134, 14310.

46. K. Dhara, Y. Hori, R. Baba, and K. Kikuchi, Chem. Commun., 2012, 48, 11534.

47. M. Minoshima, T. Matsumoto, and K. Kikuchi, Anal. Chem., 2014, 86, 7925.

48. K. Sasaki, T. Ito, N. Nishino, S. Khochbin, and M. Yoshida, Proc. Natl. Acad. Sci. U. S. A., 2009, 106, 16257. 